\title{
Régions ultrapériphériques et droit communautaires de la concurrence
}

Jean-Michel Ragald

\section{OpenEdition}

\section{Journals}

Édition électronique

URL : http://journals.openedition.org/plc/609

DOI : $10.4000 /$ plc. 609

ISSN : 2117-5209

Éditeur

L'Harmattan

Édition imprimée

Date de publication : 1 janvier 1998

Pagination : 239-273

ISSN : 1279-8657

\section{Référence électronique}

Jean-Michel Ragald, «Régions ultrapériphériques et droit communautaires de la concurrence »,

Pouvoirs dans la Caraibe [En ligne], 10 | 1998, mis en ligne le 09 mars 2011, consulté le 30 avril 2019 URL : http://journals.openedition.org/plc/609 ; DOI : 10.4000/plc.609 


\title{
Régions ultrapériphériques et droit communautaires de la concurrence
}

\author{
par Jean-Michel RAGALD \\ Doctorant en droit \\ Membre du CRPLC \\ de l'Université des Antilles et de la Guyane
}

L'analyse de l'application des règles de concurrence est essentielle pour apprécier les perspectives de développement des régions ultrapériphériques et des entreprises y exerçant des activités. A condition, toutefois, de prendre en considération des évolutions importantes intervenues dans le domaine communautaire. Le particularisme mesuré de l'application du droit communautaire de la concurrence dans les départements français d'outre-mer passait, jusqu'à la fin des années 1980, par l'appel à la généralisation des dérogations au principe de concurrence, nonobstant le principe d'application immédiate énoncé à l'article $227 \S 2$. La tendance inverse s'est amorcée, de façon séquentielle et sectorielle, par l'abandon du monopole d'Air France sur les liaisons entre la France Métropolitaine et les DOM. Une tendance identique est, depuis peu, enclenchée pour les régions insulaires du Portugal. Promesse avait été faite par le Portugal d'appliquer à ces régions, à partir du $1^{\text {er }}$ janvier 1996, le règlement $\mathrm{n}^{\circ}$ 2408/92 relatif à l'accès des transporteurs aériens communautaires aux liaisons intra-communautaires ${ }^{1}$. Parallèlement, une lecture attentive des décisions de la Commission en matière d'aides étatiques illustre, au-delà de l'importante liberté laissée à cette Institution pour les remettre en cause, sous réserve de

1. JOCE. $\mathrm{n}^{\circ}$ L. 240 du 24 août 1992, p. 8. 
l'exactitude matérielle des faits et de l'erreur manifeste d'appréciation, une assez grande indulgence.

La Cour de Justice, de son côté, n'écarte pas la possibilité d'appliquer le droit communautaire de la concurrence pour censurer éventuellement les comportements anticoncurrentiels sur le territoire de collectivités infra-étatiques ${ }^{2}$. Dès 1957, l'article $227 \S 2$ du traité $\mathrm{CE}$, concernant les départements français d'outre-mer, avait prévu l'application des dispositions communautaires du droit de la concurrence dès l'entrée en vigueur du traité de Rome. Les autres régions ultrapériphériques ne sont pas concernées par cette disposition, sauf modification éventuelle. Le droit communautaire de la concurrence s'applique, sous certaines conditions aux entreprises exerçant partiellement ou totalement leurs activités dans des régions ultramarines ainsi qu'aux collectivités régionales ou locales ellesmêmes ${ }^{3}$.

L'application des dispositions communautaires de la concurrence reçoit des aménagements du fait de la structure des marchés considérés et des conditions propres à ces règles. Comme les DOM français ${ }^{4}$, les Açores, Madère et les Canaries sont des entités régionales caractérisées par l'absence de rattachement physique au continent européen. Leur territoire respectif est exigu. La considération tenant à l'éloignement du continent européen et à la dimension de ces entités fait émerger des difficultés d'appréciation des conditions d'application des dispositions communautaires du droit antitrust et des conditions de concurrence elles-mêmes relativement aux questions d'ordre économique intéressant les régions ultrapériphériques. Un indice spécifique souligne les handicaps des entreprises ultrapériphériques ${ }^{5}$. Il intègre les charges de transport, les délais

2. Concernant les îles Canaries, CJCE 30 novembre 1995, Aff. C-134/94, Esso SA, Rec. 1995, p. I-4223.

${ }^{3}$. CJCE 14 octobre 1987, Aff. 323/82, RFA c/Conmission, Rec. 1987, p. 4013.

4. CJCE 10 octobre 1978, Aff. 148/77, Hansen, Rec. 1978, p. 1787.

5 . Sur la " situation d'ultrapériphéricité ", v. Brigitte Gaspard, "Les voies pour un renforcement de l'effet utile de l'article 227 \$2", Pouvoirs dans la Caraïbe, Rev. du CRPLC, 1997, n 8-9, p. 219. 
d'approvisionnement en matières premières et de livraison de produits finis et l'accès difficile à l'information ${ }^{6}$. Les entreprises de ces régions ne sont donc pas dans des conditions de compétition optimales.

Tout le droit communautaire de la concurrence ne s'applique pas aux régions ultrapériphériques. L'exclusion découle soit d'un seuil que n'atteignent pas les entreprises des régions ultrapériphériques, soit d'un acte explicite. Les régions ultrapériphériques ne sont pas concernées par les dispositions du traité CECA. En vertu de l'article 79 du traité de Paris, "le présent traité est applicable aux territoires européens des Hautes Parties Contractantes ". L'article 80 de ce traité ajoute que "les entreprises au sens du présent traité sont celles qui exercent une activité de production dans le domaine du charbon et de l'acier à l'intérieur des territoires visés à l'article ". Les collectivités ultramarines n'étant pas incluses dans les territoires européens, il n'y a pas lieu d'appliquer cette convention aux entreprises de ces régions, d'autant que jusqu'à une date récente aucune n'exerçait une activité visée à l'article 80. La Commission est intervenue, concernant une aide accordée en faveur de la création d'une entreprise dans l'île de la Réunion en vue de la production de gros tubes d'acier, en référence à l'encadrement de certains secteurs sidérurgiques hors $\mathrm{CECA}^{7}$.

Globalement, bien que l'application des règles communautaires de la concurrence soit limitée (I), des mesures de libéralisation, complémentaires à ces dispositions, ont suscité une ouverture de la concurrence dans certains secteurs d'activité intéressant les régions

${ }^{6}$. Jean Labasse, Quelles régions pour l'Europe?, Paris, Dominos, Flammarion, 1994, p. 105.

7. JOCE $\mathrm{n}^{\circ}$ C. 320 du 13 décembre 1988, p. 3.

Communication de la Commission, en application de l'article $93 \S 2$ du traité, adressée aux autres Etats membres et aux tiers intéressés, concernant une aide que la France a prévu d'octroyer à l'entreprise Ecopipe, un nouveau fabricant de tubes d'acier soudés de l'île de la Réunion (97/C. 125/07) ; JOCE $n^{\circ}$ C. 125 du

22 avril 1997, p. 13. Concernant une aide à finalité régionale accordée dans le cadre de l'encadrement de certains secteurs sidérurgiques hors CECA, v. 14 janvier 1997, Aff. C.-169/95, Royaume d'Espagne c./ Commission, Rec. 1997, p. I-135. 
ultrapériphériques (II). Une place toute particulière est réservée aux interventions d'Etat (III).

\section{I - L'INTENSITE MESUREE DE L'APPLICATION DES REGLES COMMUNAUTAIRES DE LA CONCURRENCE}

Outre l'hypothèse d'exclusion partielle d'application susindiquée, la mise en œuvre des règles communautaires de la concurrence est relative en raison de la difficulté à mettre en évidence une affectation du commerce entre les Etats membres (A) alors que, parallèlement, le marché pertinent traduit fréquemment une faible dimension de l'éventuelle infraction (B).

\section{A. - La condition d'affectation du commerce entre les Etats membres}

L'analyse de l'affectation du commerce entre les Etats membres, sur un territoire limité, mobilise plusieurs techniques (A). Evidemment, des éléments d'appréciation de l'existence de cette condition peuvent en justifier l'application (B).

$1^{\circ}$ - Les techniques d'analyse de l'affectation du commerce intra-communautaire

En raison de la petitesse des régions ultrapériphériques, trois techniques peuvent être relevées. Elles ne sont pas propres à ces collectivités mais elles ont un intérêt considérable pour apprécier l'applicabilité de règles communautaires de la concurrence. Il convient de noter la technique d'amplitude des effets (intégrant le seuil de sensibilité), de la structure du marché (insérant l'élimination de concurrents) et de sa dimension (économique ou géographique) ${ }^{8}$.

\footnotetext{
${ }^{8}$. La dimension du marché fera l'objet cependant d'une étude spécifique à venir.
} 
Relativement à la première technique, la notion d'effet sensible ${ }^{9}$ met en relief l'importance de l'évaluation de l'influence économique du comportement pour déterminer l'applicabilité des dispositions du droit communautaire de la concurrence ${ }^{10}$. Lorsque le marché est altéré de façon insignifiante, compte tenu de la faible position des opérateurs sur celui-ci, de la quantité limitée de produits concernés, du caractère isolé de l'accord litigieux et de la possibilité laissée à d'autres courants commerciaux de se développer pour les mêmes produits par le moyen de réexportation ou d'importation parallèle, la proscription des ententes illicites énoncée à l'article $85 \$ 1$ est écartée. Il y a peu de chance que les accords passés entre entreprises de régions ultrapériphériques aient un effet sensible. La discussion est ouverte quant à l'entente concernant des entreprises d'Etats membres distincts dont l'une, voire les deux serai(en)t ultrapériphérique(s).

En matière d'aide, la règle de minimis emporte un effet identique. Les aides accordées par des collectivités locales et régionales, dont le montant est faible, n'affectent pas les échanges entre les Etats membres. Dans le souci de simplification de la procédure, la Commission a instauré en 1992 cette règle qui autorise la fixation d'un seuil d'aide en montant absolu en dessous duquel l'article $92 \S 1$ n'est pas d'application et qui ne nécessite pas de notification. Ce montant peut être réactualisé ${ }^{11}$. La règle de minimis ne concerne pas les secteurs faisant l'objet de règles communautaires spéciales en matière d'aides d'Etat.

Atténuant la portée de la référence à l'effet sensible sur le marché pertinent, il reste également possible de tenir compte d'éléments de nature à faciliter l'établissement d'une influence directe ou indirecte sur les échanges entre les Etats membres avec un degré de

9. V. Sur l'exigence de sensibilité, CJCE 18 juin 1981, Aff. 126/80, Salonia/Poidomani et Giglio, Rec. 1981, p. 1563. Aussi, Communication de la Commission concernant les accords d'importance mineure qui ne sont pas visés par l'article $85 \S 1$ du traité CEE; JOCE $\mathrm{n}^{\circ}$ C. 231 du 12 septembre 1986, p. 2.

${ }^{10}$. CJCE 9 juillet 1969, Aff. 5/69, Völk/Vervaecke, Rec. 1969, p. 295.

11. Par exemple, en mars 1996, le seuil a été fixé à 100000 écus sur trois ans par bénéficiaire (Communication de la Commission relative aux aides de minimis ; JOCE $\mathrm{n}^{\circ}$ C. 68 du 6 mars 1996). 
probabilité suffisant ${ }^{12}$. Un comportement produisant des effets à l'intérieur du territoire d'un Etat membre relève du domaine de l'ordre juridique national ${ }^{13}$. On aurait pu dès lors conclure qu'une infraction dont les répercussions sont concentrées dans une région ultrapériphérique ne concernerait pas le droit communautaire de la concurrence, ce qui, dans son principe, n'est pas inexact. On serait alors enclin à appliquer le droit national de la concurrence, réserve faite de l'invocation simultanée du droit national et du droit communautaire de la concurrence. L'immunité d'un comportement focalisé sur une entité infra-étatique au regard du droit communautaire n'est pas évidente ${ }^{14}$. La concentration des effets à l'intérieur d'une région ultrapériphérique n'emporterait pas forcément exonération de l'application du droit communautaire de la concurrence.

La Commission a énoncé, à propos d'une aide d'Etat portant création d'une entreprise à l'île de la Réunion que la production limitée et l'éloignement du marché ciblé (en l'espèce celui des tubes soudés) pourraient minimiser, voire exclure, des effets négatifs sur les échanges entre les Etats membres de l'Union européenne, après un examen prima facie et étant donné l'état du marché. Les effets négatifs, bien qu'existants, l'emportent sur les bénéfices potentiels que la région obtiendrait de l'investissement aidé ${ }^{15}$. Le contrôle est effectué en raison de la surcapacité existant dans le secteur des tubes d'acier pour vérifier si, à moyen terme, les nouvelles capacités projetées n'auraient pas des effets négatifs en matière d'écoulement de la production qui dépasseraient les effets bénéfiques à court terme pour une région en matière de développement et d'emploi ${ }^{16}$. Les conditions

12. TPI 8 octobre 1996, Aff. T-24/93, T-25/93, T-26/93 et T-28/93, Cie Maritime belge de Transports S.A. c./ Commission, Rec. 1996, p. II-1201.

13. CJCE 31 mai 1979, Aff. 22/78, Hugin, Rec. 1979, p. 1868, point $n^{\circ} 17$. En l'espèce, les effets d'un refus de vente étaient localisés dans la région londonienne.

14. Ainsi, concernant l'application du droit communautaire de la concurrence à l'égard de comportements dont les effets concernent l'Ile-de-France, Cass. 6 mai 1996, France Télécom c./ Sté Communications Média Services, Bull. Civ., n 5, mai 1996, $\mathrm{n}^{\circ} 125$, p. 109.

${ }^{15}$. JOCE $\mathrm{n}^{\circ}$ C. 125 du 22 avril 1997, p. 13.

16. Bull. UE 4-1996, point $\mathrm{n}^{\circ} 1.3 .36$, p. 23, décision de la Commission du 30 avril 1996 en vue de l'ouverture de la procédure au titre de l'article 93 § 2 du traité CE. La 
particulières dans lesquelles se trouvent les régions ultrapériphériques, qu'elles soient économiques ou géographiques, ne sont pas de nature a écarter une potentialité d'affectation du commerce entre les Etats membres. Dans un tel cas, l'exercice est d'une délicatesse singulière.

La considération attachée à l'atteinte à la structure du marché met en exergue une présomption selon laquelle dès lors que l'on a prouvé que le comportement en cause a pour objet ou pour effet l'élimination de concurrents, il n'est pas nécessaire de démontrer que les échanges entre les Etats membres sont affectés ${ }^{17}$. Le fait d'éliminer un concurrent suffit à affecter la structure de la concurrence et affecter le commerce entre les Etats membres ${ }^{18}$. L'analyse structurelle a pleinement sa place dans un marché aussi restreint que celui d'une région ultrapériphérique. Il n'en va pas de même du recours à la théorie de la " mise en œuvre" $" 19$ qui, dans ce cadre, ne permet pas la mise en valeur d'une affectation du commerce entre les Etats membres.

\section{$2^{\circ}$ - Les éléments d'appréciation de l'existence de l'affectation du commerce entre les Etats membres}

Lorsque la Commission estime que les articles $85 \S 1$ et 86 sont inapplicables, elle délivre une attestation négative. Il a ainsi été demandé à la Commission de délivrer une attestation négative concernant des accords conclus entre les raffineries britanniques acheteuses de sucre de canne et des Etats exportateurs ${ }^{20}$.

décision finale concerne la création de l'entreprise Ecopipe, productrice de gros tubes d'acier dans l'île de la Réunion.

${ }^{17}$. CJCE 9 mars 1974, Aff. jtes 6 et 7/73, CSC et ICI c./ Commission, Rec. 1974, p. 223.

18. TPI 8 octobre 1996, Aff. T-24/93, T-25/93, T-26/93 et T-28/93, Cie Maritime belge de Transports SA c./ Commission, Rec. 1996, p. II-1201, point n ${ }^{\circ} 203$.

${ }^{19}$. CJCE 27 septembre 1987, Aff. 89/95, 104/85, 114/85, 116/85, 125/85, 127/85, 128/85 et 129/85, Ahlström c./ Commission, Rec. 1988, p. 5193.

20 Déc. Commission $n^{\circ}$ 80/183/CEE du 7 décembre 1979, “Accords pour la fourniture de sucre de canne ", point $\mathrm{n}^{\circ} 20$; JOCE $\mathrm{n}^{\circ}$ L. 39 du 15 février 1980, p. 64. 
L'appréciation de l'effet de ces accords sur le commerce intracommunautaire a été effectuée notamment en fonction de données économiques mettant en exergue l'existence d'autres sources d'approvisionnement dans des conditions favorables. Les besoins du marché étaient couverts par les betteraves cultivées, traitées et raffinées dans les Etats membres, trois Etats membres (à l'époque), à savoir le Royaume-Uni, la France et l'Italie, s'approvisionnant en sucre tiré de la canne. A la fin des années 70, la production communautaire de sucre de canne provenait des seuls départements français d'outre-mer qui fournissaient les raffineries de Nantes, de Bordeaux et de Marseille. La perspective d'un approvisionnement auprès des départements français d'outre-mer résulte du fait que "le sucre brut produit par ces départements est écoulé dans la Communauté conformément au principe de préférence communautaire et sans discrimination entre les entreprises concernées "21. En l'espèce, les ententes visaient à assurer l'approvisionnement en sucre brut destiné à être traité dans les raffineries des sociétés acheteuses au Royaume-Uni ${ }^{22}$. L'attestation négative sur la base de l'article 2 du règlement $\mathrm{n}^{\circ} 17 / 62$ a été délivrée au motif qu'aucun acheteur ne se trouve dans une situation de monopsone, l'influence que pourrait avoir un acheteur à privilégier une source d'approvisionnement plutôt qu'une autre avec pour conséquence l'élimination de fournisseurs sur le marché géographique considéré étant de ce fait annihilée.

Une entreprise nationale n'exerçant pas d'activités sur le ou les territoire(s) ultrapériphérique(s) de ce même Etat ne peut invoquer les dispositions relatives au libre établissement et par extension à la libre prestation des services en raison de l'existence d'une situation purement interne. L'éviction par une réglementation régionale de l'extension d'une activité sur le territoire d'un Etat opposable à une entreprise de cet Etat est une situation purement interne lorsque la Société ayant subi un préjudice a son siège social dans cet Etat et $\mathrm{y}$ exerce son activité ${ }^{23}$. Pour une entreprise ayant son siège social dans un Etat membre autre, les dispositions relatives au droit

\footnotetext{
21. Point $\mathrm{n}^{\circ} 4, \mathrm{a}$.

22 . Point $n^{\circ} 3$.

23 . Esso SA, point $n^{\circ} 16$.
} 
d'établissement s'appliqueraient de plein droit dès lors qu'elle souhaite étendre son activité sur une partie insulaire du territoire de l'État, même si elle exerce déjà une activité sur la partie non insulaire de l'État.

Il ne fait par contre pas de doute qu'un service entre une région ultrapériphérique d'un Etat membre et un autre Etat membre affecte le commerce entre les Etats membres. Sur ce point, l'analyse s'effectue en considération de la dimension de l'activité concernée. En matière de transports, les lignes exploitées sont individualisées par des caractéristiques particulières $^{24}$. Il n'est pas exclu que, dans la perpective de libéralisation, un marché puisse être une liaison entre une ville d'un Etat membre et une région ultrapériphérique d'un autre Etat membre. Bien évidemment, une telle situation suggère, en l'état actuel du développement des échanges, le droit des transporteurs à l'établissement de services nouveaux. De même, un comportement très fortement concentré sur un territoire restreint est susceptible d'avoir des effets sur les échanges entre les Etats membres ${ }^{25}$. L'acception originaire est importante alors que transparaît aussi l'intérêt de l'analyse du marché.

\section{B. - L'étude du marché pertinent}

La définition du marché en cause (1) permet de se faire une idée sur son importance (2).

\section{$1^{\circ}$ - La définition du marché}

Le litige Asia Motor a été l'occasion d'individualiser, dans le cadre du droit communautaire, le marché d'une région ultrapériphérique par rapport à d'autres marchés. Cette délimitation

${ }^{24}$. CJCE 11 avril 1989, Aff 66/86, Ahmed Saeed Flugreisen, Rec. 1989, p.803, point $\mathrm{n}^{\circ} 40$.

25. Déc. Commission, 4 novembre 1988, Aff. IV/32.318, European LondonSABENA ; JOCE $\mathrm{n}^{\circ}$ L. 317 du 24 novembre 1988, p. 47. 
géographique du marché restreinte à une région ultramarine est perceptible dans le droit français de la concurrence ${ }^{26}$.

Parmi les éléments de nature à particulariser le marché dans une région ultrapériphérique, il convient de citer le grand éloignement, l'insularité, l'exiguïté, la faible diversification de l'économie locale et une concurrence accrue des pays en voie de développement ${ }^{27}$. Les conditions d'approvisionnement sont inhérentes à certaines contingences, les coûts étant automatiquement augmentés en raison du transport mais aussi des besoins importants de stockage ${ }^{28}$. L'éloignement des Açores et de Madère par rapport aux sources d'approvisionnement en produits pétroliers crée une dépendance élevée de leur approvisionnement énergétique à l'égard de ces produits $^{29}$. Une dépendance externe de ces régions par rapport aux sources d'approvisionnement en produits sidérurgiques existe également $^{30}$. Comme les régions ultramarines portugaises ${ }^{31}$, les îles Canaries sont indépendantes par rapport aux sources d'approvisionnement de produits en amont de certains secteurs de l'alimentation essentiels à la consommation courante ou à la

26. Deux entreprises pétrolières propriétaires d'installations de stockage de carburéacteurs de l'aéroport de Saint-Denis de la Réunion disposaient ensemble d'une position dominante sur le marché de ce produit dans le département de la Réunion $(\mathrm{C}$. App. Paris, 6 juillet 1994, RJDA 12/94 n 1324). De même, le Conseil de la concurrence admet la possibilité d'infraction sur un marché uniquement cantonné à un DOM (Déc. $\mathrm{n}^{\circ}$ 96.D.63 du 26 octobre 1996, BOCCRF du 8 janvier 1997). Globalement, sur la délimitation du marché géographique en droit français de la concurrence, Marie Malaurie-Vignal, Droit interne de la concurrence, Armand Colin, Ed. 1996, p. 194.

${ }^{27}$. Elisa Paulin et Marie-Josèphe Rigobert, "Les régions ultrapériphériques et la CEE", Rev. March. Comm. et de l'Un. Eur. n 368, mai 1993, p. 436.

${ }^{28}$. Pedro Burgos, “Iles Canaries et les Communautés européennes", Rev. March. Comm. $n^{\circ}$ 302, décembre 1986, p. 580 et s. V. aussi déc. Conseil, 26 juin 1991, 91/315/CEE, POSEIMA point 10.3, JOCE $\mathrm{n}^{\circ}$ L. $171 \mathrm{du} 29$ juin 1991, p. 10.

${ }^{29}$. Déc. POSEIMA, cons. $n^{\circ} 14.1$ (31 Déc. POSEIMA, cons. $n^{\circ} 13$.

${ }^{30}$. Déc. POSEIMA, cons. $\mathrm{n}^{\circ} 16$.

31 . Déc. POSEIMA, cons. $n^{\circ} 13$. 
transformation ${ }^{32}$. Cela est dû à la "situation géographique exceptionnelle ${ }^{, 33}$.

\section{$2^{\circ}$ - L'importance du marché}

Au titre de l'article 86 du traité CE, la question se pose de savoir si une entité sub-étatique peut constituer une partie substantielle du marché commun. La Cour de Justice des Communautés européennes a eu l'occasion de se prononcer à ce sujet mais elle l'a quelque peu contournée $^{34}$. Le comportement d'une entreprise en position dominante sur un marché régional ne relève pas de l'interdiction posée à l'article $86 \mathrm{du}$ traité CE dans la mesure où elle n'est pas dominante sur un marché géographiquement plus vaste pris en compte par la Cour de Justice. L'examen de la possibilité qu'une production d'une région ultrapériphérique ou qu'une installation s'y trouvant représente une partie substantielle du marché commun suppose au préalable la " singularisation" du marché régional. L'existence d'une partie substantielle du marché commun tient plus à la dimension économique du territoire considéré qu'à son étendue. De ce fait, une entité infraétatique pourrait avoir cette qualité. Selon la Cour, " en vue d'établir si un territoire déterminé revêt une importance suffisante pour constituer une partie substantielle du marché commun, il faut prendre en considération, notamment, la structure et le volume de la production et de la consommation dudit produit, ainsi que les habitudes et les possibilités économiques des vendeurs et des acheteurs " ${ }^{35}$. L'appréciation du poids économique des collectivités sub-étatiques ne peut être réalisée sans référence à la population, à la localisation des produits voire aux possibilités d'écoulement de ceux-ci. En raison des

${ }^{32}$. Déc. Conseil, 26 juin 1991, 91/3141/CEE, POSEICAN, cons. $n^{\circ} 7$; JOCE $\mathrm{n}^{\circ}$ L. 171 du 29 juin 1991.

33. Pour les îles Canaries, cons. $n^{\circ} 7$ de la décision POSEICAN.

${ }^{34}$. CJCE 5 octobre 1988, Aff. 247/86, Alsatel/Novasam, Rec. 1988, p. 5987

${ }^{35}$. CJCE 16 décembre 1975 AS. jtes 40 à 48, 50, 54 à 56, 111, 113 et 114/73, Suiker, point $\mathrm{n}^{\circ} 371$, Rec. 1975 , p. 1663. 
conditions structurelles et géographiques particulières, une région ultrapériphérique ne peut représenter une partie substantielle du marché commun, sous réserve de certaines productions majeures insusceptibles d'être développées sur d'autres parties du territoire communautaire.

Sur le plan infrastructures, des installations essentielles dans chacune de ces régions ne constituent pas une partie substantielle du marché commun compte tenu du volume du trafic dans celles-ci et de l'importance qu'elles revêtent pour l'ensemble des importations et exportations ou des entrées et sorties de personnes sur le territoire infra-étatique ${ }^{36}$. Le marché étant restreint, la référence à l'importance de l'aire géographique est peu pertinente. La possibilité d'empêchement de la pénétration de produits ou de services sur ce marché restreint, encore qu'il puisse être individualisé, est la méthode la mieux adaptée aux contingences particulières sus-indiquées.

\section{II. - L'OUVERTURE DE LA CONCURRENCE DANS LES REGIONS ULTRAPERIPHERIQUES}

Malgré la mise en place de mécanismes en vue de promouvoir l'ouverture de la concurrence (A), les obligations de service public doivent être respectées (B).

36. Cette position peut paraître audacieuse quand on sait que la Cour de Justice s'est prononcé sur la qualité de partie substantielle du marché commun par référence à l'importance économique de celle-ci vis-à-vis de l'État membre et non vis-à-vis d'une entité locale ou régionale (CJCE. 10 décembre 1991, Aff. C-179/90, Port de Gênes 1, Rec. 1991, p. I-5889 ; CJCE 17 mai 1994, Aff. 18/93, Corsica Ferries, Rec. 1994, p. I-1783). En réalité, tout dépend de l'activité et/ou du produit concerné. Les bananes étant produites uniquement dans les régions ultrapériphériques, le transport maritime de bananes en provenance des ports de ces régions fait de ceux-ci, vis-à-vis de leur Etat respectif, une partie substantielle du marché commun. Ce raisonnement n'est pas extensible à toutes les activités développées au sein de ces installations. Rappelons par ailleurs que l'aéroport de Bruxelles, intégré comme les aéroports des DOM dans les réseaux transeuropéens de transport, constitue une partie substantielle du marché commun (Déc. Commission n ${ }^{\circ}$ 95/364/CE du 28 juin 1995, RVA ; JOCE $\mathrm{n}^{\circ}$ L. 216 du 12 septembre 1995, p. 8). 


\section{A. - Les mécanismes de promotion de la concurrence dans les régions ultrapériphériques}

On peut réfléchir sur deux aspects de la question, le comportement tarifaire d'une entreprise disposant d'un monopole (1) et la problématique de l'accès au marché local (2).

\section{$1^{\circ}$ - Le comportement tarifaire du monopoleur}

Le monopole de la CGM sur la ligne maritime desservant les Antilles a eu pour conséquence la fixation de prix élevés affectant le marché des exportations, ce qui est de nature à engendrer l'augmentation des prix des produits originaires des DOM par rapport aux produits concurrents, surtout provenant d'Etats tiers de l'Union européenne, et qui se trouvent, sur ce plan notamment, favorisés. Pour l'instant, le maintien du monopole peut s'expliquer par le caractère déficitaire des lignes desservies en partance ou à destination des DOM $\mathrm{Au}$ demeurant, malgré les allégations du gouvernement britannique et de certains concurrents de la CGM, notamment pour les lignes Antilles, la Commission n'a pas jugé nécessaire d'effectuer des investigations au titre de l'article 86 du traité CE étant donné qu'elle ne dispose pas de suffisamment d'informations ${ }^{37}$.

La démonopolisation préconisée peut avoir un effet interne sur la concurrence dans une région ultrapériphérique. Un transporteur aérien, en situation monopolistique sur la ligne Paris-Antilles, avait décidé d'augmenter le tarif du fret. Entre deux entreprises installées dans un DOM, concurrentes sur le marché de l'aviculture, l'un produisant ses propres œufs et l'autre les achetant en France métropolitaine, l'augmentation du tarif du fret a pour conséquence de favoriser l'entreprise produisant ses propres œufs. Le comportement de

37. Déc. Commission, 17 juillet 1996 concernant l'aide accordée à la Compagnie Générale Maritime dans le cadre d'un plan de restructuration, Aff. 97/14/CE, point $\mathrm{n}^{\circ} \mathrm{IX}, \mathrm{ii} ;$ JOCE $\mathrm{n}^{\circ} \mathrm{L} .5 \mathrm{du} 9$ janvier 1997. 
ce transporteur produisait des effets sur un marché dans lequel aucun intérêt personnel n'est à relever ${ }^{38}$.

Dans la mesure où certains monopoles sont maintenus, à l'exemple de celui des Chambres de Commerce et d'Industrie sur les aéroports, l'atténuation des excès tarifaires en imposant le respect du principe de relation avec les coûts ${ }^{39}$ est recherchée.

\section{$2^{\circ}$ - L'accès au marché}

Une question importante est celle de savoir si le fait de permettre la pénétration d'une entreprise d'un autre Etat membre sur le territoire d'un Etat sans qu'elle ne soit autorisée à accéder au marché ultrapériphérique constitue une atteinte aux dispositions du droit communautaire de la concurrence. L'analyse de la faculté de l'entreprise concurrente à exercer son activité serait déterminante. Si l'accès à la région ultrapériphérique est une condition d'existence de l'activité elle-même, la dimension de l'activité ou du marché faciliterait le recours aux dispositions communautaires pertinentes. $\mathrm{Si}$ l'activité dans la région ultrapériphérique ne conditionne pas l'exercice de l'activité dans la métropole, la capacité de l'entreprise à opérer dans ladite région et la structure du marché local sont des indices à prendre en compte.

L'ouverture des infrastructures à la concurrence ne peut se faire du côté des régions ultrapériphériques, que selon la vision particulière de la bottleneck doctrine. L'admission d'entreprises dans les aéroports incontournables a permis le démantèlement de situations monopolistiques. Ainsi, l'accès de transporteurs aériens concurrents nécessitait l'accès à chaque installation essentielle des Régions d'outre-mer. Un règlement amiable du 30 octobre 1990 entre la Commission des Communautés européennes, les autorités françaises et

${ }^{38}$. Cass. Comm. 19 février 1991, n 89-14.517, Bull. Civ. IV, $\mathrm{n}^{\circ}$ 82, p. 55.

39 . Proposition de directive du Conseil concernant les redevances aéroportuaires présentée par la Commission à Bruxelles le 23 avril 1997, COM (97) 154 final. 
le groupe Air-France est intervenu pour qu'au moins une compagnie établie en France desserve certaines routes intérieures.

L'éviction du monopole en matière de transports aériens a été d'autant plus aisée que l'exclusion des DOM de la décision $87 / 602^{40}$ n'a pas été reprise dans la réglementation de $1990^{41}$ et de 1992 , allant dans le sens de 1 'objectif de libéralisation de transports aériens contenu dans la décision POSEIDOM.

Des transporteurs communautaires non français peuvent exploiter des lignes reliant une ville dans la Communauté non française à une région des DOM. La ligne Bonn-Fort-de-France souhaitée par la Lufhtansa n'a pas été ouverte. De son côté, la compagnie aérienne Minerve avait demandé à assurer un vol hebdomadaire Bruxelles-La Réunion $^{42}$. Il y eut un avis favorable du Conseil supérieur de l'Aviation marchande en date du 27 octobre 1982. Mais, suite aux réticences du ministre des Transports de l'époque, des négociations entre Minerve et le Groupe Air France ont eu lieu pour tenter d'organiser une concurrence pour cette ligne. Minerve s'était aussi vu ouverte la desserte Bruxelles-Pointe-à-Pitre dont les vols étaient commercialisés par l'organisme de voyages Nouvelles Frontières. Le règlement $\mathrm{n}^{\circ} 2408 / 92$ est entré en vigueur dans les DOM le $1^{\mathrm{er}}$ janvier 1993. Le droit de cabotage total a été libéralisé le $1^{\mathrm{er}}$ avril 1997. En matière de cabotage maritime, les DOM sont temporairement exemptés du principe de libre circulation des services aux transports maritimes à l'intérieur des Etats membres ${ }^{43}$. Cette dérogation est accordée jusqu'au

40. JOCE $\mathrm{n}^{\circ}$ L. 374 du 31 décembre 1987, p. 19.

${ }^{41}$. Règl. n 2343/90 du 24 juillet 1990 concernant l'accès des transporteurs aériens aux liaisons intra-communautaires et la répartition de la capacité des sièges sur les services aériens réguliers entre les Etats membre, JOCE $\mathrm{n}^{\circ}$ L. 217 du 11 août 1990, p. 8 .

42. Michel De Juglart, Traité de Droit aérien, Tome I, 1989, spéc. p. 191.

${ }^{43}$. Règl. (CEE) n 3577/92 du Conseil du 7 décembre 1992 concernant l'application du principe de libre circulation des services aux transports maritimes à l'intérieur des Etats membres, JOCE $\mathrm{n}^{\circ}$ L. 364 du 12 décembre 1992. 
$1^{\text {er }}$ janvier 1999, les contrats de service public existants pouvaient rester en vigueur jusqu'à leur expiration ${ }^{44}$.

Par ailleurs, les aéroports des DOM sont intégrés dans les réseaux transeuropéens de transport ${ }^{45}$. L'action de la Communauté en la matière doit s'effectuer "dans le cadre d'un système de marchés ouverts et concurrentiels" en favorisant notamment "l'accès à ces réseaux " 46 . Les régions ultrapériphériques sont fortement intéressées par "la nécessité de relier les régions insulaires, enclavées et périphériques aux régions centrales de la Communauté "47.

\section{B. - Le service public dans les régions ultrapériphériques}

Intéressant les régions ultrapériphériques, la politique d'aménagement du territoire et du développement économique et social d'entités infra-étatiques est intégrée dans les exigences de service public avec l'impératif de réaliser des programmes particuliers en matière d'équipements ${ }^{48}$.

Un Etat doit être libre de déterminer les modalités et la substance du service public, y compris dans une région ultrapériphérique. Il intervient de diverses façons. Son intervention peut ainsi prendre la forme d'une aide. Lorsque la compensation au titre du service public a pour seule fonction de financer les coûts des

44. Rapport 1992-1993 sur les progrès accomplis dans la mise en œuvre du POSEIDOM présenté par la Commission au Conseil et au Parlement européen conformément au titre $\mathrm{V}$ de la déc. $\mathrm{n}^{\circ}$ 89/687/ CEE et de l'article 23 du règl. (CEE) $\mathrm{n}^{\circ}$ 3763/91, COM (94) 200 final, Bruxelles, le 30 mai 1994, point $\mathrm{n}^{\circ} 88$.

45 . Dir. $n^{\circ}$ 1692/96 du Parlement européen et du Conseil du 23 juillet 1996 sur les orientations communautaires pour le développement du réseau transeuropéen de transport; JOCE $\mathrm{n}^{\circ}$ L. 228 du 9 septembre 1996, p. 1.

${ }^{46}$. Art. $129 \mathrm{~B} \S 2$ du traité CE.

47. Art. 129 B $\$ 2$ du traité CE.

48. TPI 27 février 1997, Aff. 106/95, FFSA et autres c./ Commissions point $n^{\circ} 70$, non publié. 
obligations de service public sous peine d'être interdite sur la base de l'article $92 \S 1^{49}$.

En vertu de l'article $4 \S 1$ du règlement $n^{\circ} 2408 / 92$ du 23 juillet 1992, un Etat membre peut, à la suite de consultations avec les autres Etats membres concernés et après en avoir informé la Commission et les transporteurs aériens qui exploitent la liaison, imposer des obligations de service public sur des services aériens réguliers vers un aéroport desservant une zone périphérique ou de développement située sur son territoire. L'imposition de l'obligation de service public est admise pour les liaisons considérées comme vitales pour le développement économique de la région dans laquelle est situe l'aéroport. Le principe de proportionnalité veut qu'elle vise à assurer sur une liaison une prestation de services adéquate répondant à des normes fixes en matière de continuité, de régularité, de capacité et de prix, normes auxquelles le transporteur ne satisferait pas s'il ne devait considérer que son seul intérêt commercial.

Les conditions d'imposition de l'obligation de service public dépendent de la localisation de l'installation de destination, qui doit être située dans une zone périphérique ou de développement. La volonté d'assurer le transport d'usagers habitant dans la zone en question naît du souci de relier l'ensemble du territoire de l'Etat, y compris les zones les plus reculées ${ }^{50}$. L'obligation d'exploitation des lignes non rentables du point de vue commercial, nécessaires pour des raisons d'intérêt général, est un indice en vue de permettre l'application de l'article $90 \S 2^{51}$. Les obligations de service public sont imposées par l'Etat " en termes de fréquences minimales, de types d'appareils utilisés et de capacité offerte, d'horaires, de politique commerciale ou de continuité du service ". L'exigence de continuité

${ }^{49}$ Déc. Commission des 2 et 9 octobre 1996 relative aux financements publics accordés à la Chaîne de TV publique portugaise RTP, Bull. UE 10-1996, n 1.3.61, p. 32.

${ }^{50}$. Pour une application de l'article $4 \S 1$, a) du règl. $n^{\circ} 2409 / 92$ aux régions reculées, v. l'obligation de service public imposée à la société Transportes Aeros Portugueses pour l'exploitation des lignes à destination des régions autonomes portugaises des Açores et Madère (JOCE ${ }^{\circ}$ L. 260 du 8 octobre 1994, p. 77).

51. CJCE 11 avril 1989, Aff. 66/86, Aff Ahmed Saeed Flugreisen, Rec. 1989, p. 803. 
impose un nombre de fréquences minimales et des horaires plus ou moins constants, mais aussi un faible pourcentage du nombre de vols annulés.

Les régions ultramarines disposent d'aéroports constituant un instrument important de leur développement. Toutefois, la situation des DOM par rapport aux autres régions périphériques de l'Union européenne est différente. Une très forte demande en direction de ces îles entraîne un trafic important, réserve faite du cas guyanais ${ }^{52}$. Malgré une localisation et une importance des installations, l'introduction du service public dans ces régions en matière de transport aérien n'intègre pas la nécessité d'exploiter des lignes non rentables, la structure de la demande permettant l'introduction de la concurrence pour le transport aérien de personnes.

Le cas des régions insulaires portugaises est distincte de celui des DOM. Une compensation du déficit dû à la limitation par une entreprise nationale de l'accès à une région ultrapériphérique constitue une aide accordée au transporteur. Cependant, tant que les mesures de libéralisation prévues par le règlement $n^{\circ}$ 2408/92 concernant l'accès des transporteurs aériens communautaires aux liaisons intracommunautaires n'étaient pas applicables sans restrictions, les compensations au titre d'obligations de service public pouvaient être acceptées sur la base de l'article $92 \S 3$ point a) du traité CE et de l'article $61 \S 3$ point a) de l'accord EEE. La Commission justifie sa position sur l'appréciation du “degré d'ouverture de l'accès au marché " pour déterminer si la compensation du déficit de l'entreprise nationale opérant en aval est le seul moyen de maintenir les liaisons d'importance vitale pour ces régions. Tant que l'accès à ces lignes n'est pas entièrement libéralisé, le seul moyen pour un Etat de faire face aux graves problèmes économiques et sociaux liés à l'éloignement d'îles consiste à imposer une obligation de service public pour la desserte de ces îles et à compenser le déficit enregistré par l'entreprise disposant du monopole sur ces lignes. Le contexte a, pour les régions

52. V. communication au titre de l'article $1 \S 4$ de la dir. ${ }^{\circ}$ 96/67/CE du Conseil (97/C. 117/09) ; JOCE $\mathrm{n}^{\circ}$ C. 117 du 15 avril 1997, p. 13. 
autonomes du Portugal, changé - $^{53}$ depuis le $1^{\text {er }}$ janvier 1996, avec l'application du règlement $\mathrm{n}^{\circ} 2408 / 92$, la libéralisation étant déjà admise dans les DOM. Jusqu'au $1^{\text {er }}$ janvier 1996, en ce qui concerne les régions ultrapériphériques portugaises, un régime d'aide destiné à compenser un déficit résultant pour l'entreprise nationale des obligations de service public sur les liaisons avec les régions de Madère et des Açores était compatible avec le marché commun dans la mesure où cette aide est destinée à favoriser le développement des régions dans lesquelles le niveau de vie est anormalement bas ou dans lesquelles sévit un grave sous-emploi, à condition que le montant de l'aide octroyée ne soit pas supérieur au déficit enregistré sur les lignes. Certaines communications ont imposé des obligations de service public relatives à la desserte des îles, parmi lesquelles on trouve les Açores pour le Portugal ${ }^{54}$. L'obligation de service public intègre notamment les différenciations tarifaires ${ }^{55}$. Sans que les mesures prises soient spécifiques aux régions ultrapériphériques ${ }^{56}$, les communications les concernant dénotent l'intérêt de la prise en compte des difficultés résultant de la situation insulaire ${ }^{57}$.

Dans le domaine du transport de marchandises, la nature du marché est telle que la concurrence intermodale est inexistante ${ }^{58}$ alors que, parallèlement, le transport par navire est subordonné à une

53. Jean-Pierre Hache, "The implications of European Union legislation upon the provision of transport services with regards to Islands regions" in Systems of the Islands, L'Harmattan, p. 11.

${ }^{54}$. Communication du 4 août 1995, JOCE $n^{\circ}$ L. 200, p. 3-8.

55 . Loïc Grard, "Les obligations de services publics et le transport aérien ”, Colloque Cedece, Irène, Strasbourg, 17-19 octobre 1996, Service public et Communauté européenne : entre l'intérêt général et le marché.

56. Le Conseil d'Etat s'est notamment prononcé sur le cas de l'Ile du Ré (CE.

10 mai 1974, Denoyez et Chorques, Rec. Lebon. 274).

57. Dans la communication du 4 août 1995 concernant les Açores, une réduction tarifaire aux résidents ou étudiants poursuivant leurs études dans les îles a été envisagée.

58 . Euristes, Systèmes de transport dans les îles, L'Harmattan, Ed. 1996, p. 18. 
véritable obligation de service public avec versements de subventions compensatoires 59 .

\section{III. - L'INTERVENTION DE L'ÉTAT MEMBRE DANS LES REGIONS ULTRAPERIPHERIQUES}

Il convient de distinguer les aides d'états membres (A) et les comportements étatiques déterminant le cadre d'action d'entreprises exerçant leurs activités dans des régions ultrapériphériques (B).

\section{A. - Le contrôle effectué sur les aides d'Etats membres}

Il existe plusieurs catégories d'aides. Retiendront notre attention les aides de fonctionnement et les aides à l'investissement ou à la création d'emplois (1), les aides à la sauvegarde et à la restructuration d'entreprises opérant dans des "régions assistées" (2) et la fiscalité spécifique (3). La tendance générale est à la mansuétude. La perspective dérogatoire en la matière résulte d'une démarche à la fois contextuelle et téléologique.

$1^{\circ}$ - La distinction entre les aides de fonctionnement et

les aides à l'investissement ou à la création d'emplois

Pour justifier l'application de l'article $92 \S 3$ sous $a$, il faut distinguer deux catégories d'aides, celles qui sont liées à un investissement initial ou à la création d'emploi et les aides au fonctionnement à caractère continu visant à surmonter des handicaps particuliers ou permanents.

La Commission admet les aides au fonctionnement sous certaines conditions. L'aide doit être limitée dans le temps et avoir

59. Françoise Odier, Transports maritimes, orientations générales, J-Cl. Europe, Fasc. 1140, 5, 1994, p. 18. 
pour objet de surmonter les handicaps structurels d'entreprises implantées dans les régions proposées au titre de l'article 92 § 3 littera $a^{60}$. Elle doit viser à promouvoir le développement durable et équilibré de l'activité économique et ne pas susciter des surcapacités sectorielles au niveau communautaire, de telle sorte que le problème sectoriel créé sur le plan de la Communauté serait plus grave que le problème régional initial. Il serait particulièrement étonnant que les problèmes économiques localisés dans les régions ultramarines puissent s'étendre à la Communauté. L'aide de fonctionnement ne doit pas être accordée en violation des règles spécifiques visant les aides consenties aux entreprises en difficulté. Les aides visant à promouvoir les exportations vers d'autres Etats membres sont à exclure.

Concernant les aides liées à un investissement initial ou à la création d'emplois, les Etats membres peuvent prendre des dispositions de "défiscalisation" $" 61$ applicables uniquement dans ces régions et de nature à subir l'examen de la Commission. Une disposition nationale de " défiscalisation " propre aux DOM n'est pas contraire à l'article 92 au double motif tiré de la territorialité et du développement. Si la mesure discrimine les entreprises étatiques (y compris locales) par rapport à d'autres entreprises, elle constitue une aide interdite. Il en est de même si les entreprises locales sont privilégiées par rapport aux entreprises d'autres Etats membres. Le principe de territorialité impose que, dans ces régions, l'aide doive être applicable à toutes les entreprises situées sur le territoire et à toutes celles désireuses de s'insérer dans le marché local. L'entreprise bénéficiant du droit de déduction au titre des investissements directs, relativement à la loi Pons, lorsque l'activité concerne l'industrie, la pêche, 1'hôtellerie, le tourisme, les énergies nouvelles, l'agriculture, le bâtiment et les

${ }^{60}$. Les régions ultrapériphériques ne sont pas les seules à bénéficier de la dérogation au principe d'interdiction des aides visées à l'article $92 \S 3$ littera $a$. Est, entre autres, également visé par les aides au fonctionnement l'ensemble constitué de la Grèce, du Portugal de l'Irlande ainsi que de l'Irlande du Nord. A propos des îles Canaries, seules sont concernées Las Palmas et Ténériffe.

${ }^{61}$. Un dégrèvement ou une exonération fiscale constitue une aide au sens de l'article 92 (CJCE 2 juillet 1974, Aff 173/73, République italienne c./ Commission, Rec. 1974, p. 709). 
travaux publics, les transports ou l'artisanat, doit être locale. Cela concerne l'exploitation d'un établissement situé dans un DOM mais aussi l'entreprise ayant son domicile ou son siège social en France métropolitaine qui y exerce des activités et y développe parallèlement des exploitations situées dans le DOM ${ }^{62}$. La loi est non-discriminatoire car les entreprises étrangères pourraient réaliser une implantation dans les DOM soit par la création d'une filiale, soit par la mise en place d'une succursale. L'assujettissement à l'impôt sur les sociétés de la succursale pourrait souffrir d'une déduction sur son bénéfice imposable par la prise en compte de 1" investissement productif" outre-mer, dans des conditions identiques à l'activité similaire d'une entreprise domiciliée ou siégeant en France métropolitaine.

Un DOM, comme l'île de la Réunion, lieu d'implantation de l'entreprise Ecopipe, pâtit d'un taux très élevé de chômage $(40 \%)$ et les activités industrielles y sont pratiquement inexistantes ${ }^{63}$. Sa localisation géographique, à plus de 10000 kilomètres du territoire métropolitain, est la principale cause de ses difficultés économiques, le projet représentant par ailleurs le premier investissement étranger dans l'île. L'aide d'Etat à la création de l'entreprise Ecopipe correspond à $34 \%$ du financement total de la création de 1'entreprise, soit 16,24 millions de Francs ${ }^{64}$, $10 \%$ du financement étant effectué par le FEDER. Basant son raisonnement sur le principe de l'investisseur privé ${ }^{65}$ et sur l'absence éventuelle de viabilité de l'entreprise, la Commission a, outre la situation sectorielle, tenu compte "dans son évaluation, de la dimension régionale des investissements aidés et, en particulier, de la situation spécifique de l'île de la Réunion conformément au point $\mathrm{n}^{\circ}$ 10.2 du titre II de la décision POSEIDOM. Dans le cas où l'entreprise bénéficiaire de l'aide est implantée dans une région assistée, en l'espèce une région ultrapériphérique, il convient de mettre en balance

${ }^{62}$. Bernard Castagnède, La défiscalisation des investissements d'outre-mer, Paris, PUF, Ed. 1987.

63. JOCE $\mathrm{n}^{\circ}$ C. 125 du 22 avril 1997, p.13.

${ }^{64}$. Bull. UE 12-1996, point $\mathrm{n}^{\circ} 1.3 .83$.

65. CJCE 2 mars 1991, Aff. 305/89, Italie c./ Commission, Alfa Roméo, Rec. 1991, p. 1603, point $\mathrm{n}^{\circ} 24$; CJCE 21 mars 1991, Aff. 303/88 Italie c/ Commission, Rec. 1991, p. 1433, point $n^{\circ} 20$; CJCE 14 septembre 1994, Aff. 42/93, Espagne c./ Commission, Rec 1994, p. I-4175, point $n^{\circ} 14$. 
les effets bénéfiques sur le développement régional susceptible de résulter des investissements aidés avec leurs éventuels effets négatifs sur la concurrence". La balance est donc faite entre les avantages sur le plan du développement régional et des conséquences préjudiciables éventuelles sur l'ensemble du secteur des tubes soudés dans le cas d'espèce. A ce titre, la Commission donne priorité à la situation régionale, rappelant que l'île de la Réunion est classée dans la liste des régions visées à l'article $92 \S 3$ point $a$ du traité, lui permettant ainsi de bénéficier du plafond d'intensité d'aide maximal au niveau communautaire. Quoique le produit soit surcapacitaire au niveau de l'Union européenne, son impact éventuel sur la concurrence et les échanges intra-communautaires reste faible car l'entreprise bénéficiaire est une PME dont la production est marginale par rapport à la production communautaire. L'aide peut être justifiée par un intérêt commun caractérisé par le développement régional. Outre la situation de l'emploi, l'effet bénéfique sur l'environnement (recyclage des plastiques collectés dans les ordures ménagères à la Réunion) a également été pris en considération. L'aide a été approuvée au titre de l'article $92 \S 3$ point $a$, avec la réserve que les autorités françaises transmettent à la Commission les comptes annuels de l'entreprise et un rapport annuel sur l'exécution du projet pendant cinq ans à compter du début de fonctionnement de l'entreprise Ecopipe, avec indication notamment, des aides payées, de l'évolution du personnel, de la production de l'entreprise et des exportations par pays de destination.

Les aides destinées à favoriser le développement économique de régions dans lesquelles le niveau de vie est anormalement bas ou dans lesquelles sévit un grave sous-emploi entre dans la catégorie des aides facultatives mentionnées à l'article $92 \$ 3$ du traité CE et qui sont, dans une large mesure, laissées à l'appréciation de la Commission ${ }^{66}$. Selon la Cour, " lorsqu'un programme d'aides à finalité régionale remplit les conditions de l'article $92 \S 1$ du traité, il importe alors de vérifier dans quelle mesure il peut bénéficier de l'une des dérogations prévues à l'article $92 \S 3 a$ et $c$ du traité. A cet égard, l'emploi des termes "anormalement" et "grave" dans la dérogation contenue dans la

${ }^{66}$. CJCE 17 septembre 1980, Aff. 730/79, Philip Morris, Rec. 1980, p. 2671. 
lettre $a$ montre que celle-ci ne concerne que les régions où la situation économique est extrêmement défavorable par rapport à l'ensemble de la Communauté. En revanche, la dérogation prévue dans la lettre $c$ est plus large, en ce qu'elle permet le développement de certaines régions sans être limitée par les conditions économiques de la lettre $a$, pourvu que les aides qui y sont dispensées n'altèrent pas les échanges dans une mesure contraire à l'intérêt commun. Cette disposition donne à la Commission le pouvoir d'autoriser les aides destinées à promouvoir le développement économique des régions d'un Etat membre qui sont défavorisées par rapport à la moyenne nationale "67. L'objectif de développement économique implique que les entreprises implantées doivent faire face à des coûts supplémentaires en raison de leur localisation et des déficiences en équipements pesant en permanence sur leur compétitivité.

L'insularité, sans être le seul argument, constitue pour un certain nombre d'aides une justification forte de l'indulgence de la Commission. La différence de formulation des lettres $a$ et $c$ de l'article $92 \S 3$ du traité ne saurait conduire à considérer que la Commission doive tenir compte de l'intérêt communautaire lorsqu'elle fait application de la lettre $a$ dudit article et qu'elle doive se borner à vérifier la spécificité régionale des mesures en cause sans évaluer leur incidence sur le ou les marchés pertinents de l'ensemble de la Communauté $^{68}$. La politique de crédit de l'Institut d'Emission des $\mathrm{DOM}^{69}$ en faveur des entreprises s'effectue par des aides d'Etat destinées à favoriser le développement économique des DOM et bénéficiant de la dérogation de l'article $92 \S 3$ point a malgré

${ }^{67}$. CJCE 14 octobre 1987, Aff. 248/84, RFA c./ Commission, Rec. 1987, p. 401.

68. CJCE 14 janvier 1997, Aff. C-169/95, Royaume d'Espagne c./ Commission, point $\mathrm{n}^{\circ} 17$, Rec. 1997, p I-135.

69. Il s'agit d'un organisme public intervenant en délivrant au cas par cas des accords de réescompte ou de classement pour les concours à court ou moyen terme accordés aux entreprises des DOM. Les accords de réescompte permettent de refinancer à des taux préférentiels les crédits consentis à certaines entreprises des DOM dans certains secteurs d'activité. Grâce à ces accords, les établissements de crédits obtiennent un financement à taux réduit assuré par l'IEDOM (aide de fonctionnement). De même, ils permettent aux entreprises des DOM de diminuer les coûts de certains de leurs emprunts (aide au fonctionnement). 
l'importance des montants alloués et leur intensité élevée. Les principaux bénéficiaires de ces aides sont les PME-PMI.

Dans une décision de la Commission du 4 mai 1994 relative au fonds de garantie en faveur des entreprises des DOM géré par la Société de gestion de ce fonds, à savoir la SOFODOM, l'indulgence de la Commission au profit de ce régime d'aide tenait à la fois à la modicité des aides, au faible développement des DOM et à leur situation ultrapériphérique ${ }^{70}$.

La Commission a eu aussi à examiner des régimes d'aides fiscales parmi lesquels on peut citer, pour l'année 1992, les dispositions de l'article 208 quater-I du code général des impôts concernant le régime d'exonération temporaire d'impôt sur les sociétés des entreprises créant une activité nouvelle dans les DOM, la réduction d'assiette pour les bénéfices réalisés outre-mer, mentionnée à l'article 207 bis de ce même code, le régime fiscal de longue durée dans les DOM figurant à l'article 1655 de ce code et le régime d'incitation fiscale contenu dans la loi Pons.

Dans une décision du 4 décembre $1996^{71}$, la Commission a fait preuve d'une mansuétude mesurée, en adoptant une décision partiellement négative à propos des aides directes en faveur de l'investissement dans les DOM et destinées à la construction de navires. Elle a exigé que la loi Pons sur la défiscalisation ne s'applique pas aux navires de plus de 100 tonnes brutes ${ }^{72}$. Ainsi, dans le cas où les aides entrent dans le champ d'application de la directive 90/684, elles sont incompatibles avec le marché commun lorsqu'elles s'appliquent à des contrats relatifs à la construction de navires destinés aux DOM mais qui

${ }^{70}$. Bull. UE 5-1994, p. 24, point 1.2.35.

${ }^{71}$. Bull. UE 12-1996, p. 65, point 1.3.86.

${ }^{72}$. Ce seuil n'est pas anodin, puisque la directive (CEE) $n^{\circ} 90 / 684 \mathrm{du}$ Conseil du 21 décembre 1990, concernant les aides à la construction navale, en son article $1^{\text {er }}$ a) définit la construction navale comme la construction à coque métallique de navires de commerce pour le transport de passagers ou de marchandises, d'au moins 100 tonnes brutes, de bateaux de pêche d'au moins 100 tonnes brutes et de dragues ou autres navires pour travaux en mer, d'au moins 100 tonnes brutes, à l'exclusion des platesformes de forages. 
sont construits dans des chantiers communautaires de 1'Union. La Commission n'a pas cependant soulevé d'objection à l'encontre des aides qui avaient été octroyées dans les cas particuliers de cinq navires, eu égard aux caractéristiques spécifiques de chacun des contrats. De même, à la suite de l'examen du projet d'extension de certaines aides au titre de la loi Pasqua ${ }^{73}$ aux DOM $^{74}$, l'ajout : de certaines aides supplémentaires pour ces régions a fait 1'objet d'un examen poussé. Il fut demandé au Ministère des DOM de veiller à ce que les aides ne dépassent pas un certain montant.

\section{$2^{\circ}$ - Les aides à la restructuration d'entreprises opérant dans des "régions assistées "}

Le contrôle des aides à la restructuration d'entreprises opérant dans des "régions assistées" ne concerne pas seulement les régions ultrapériphériques ${ }^{75}$. En vertu des lignes directrices communautaires pour les aides d'Etat au sauvetage et à la restructuration des entreprises en difficulté, les aides à la restructuration des entreprises opérant dans les " régions assistées" doivent être appréciées par la Commission en tenant compte des besoins du développement régional, et ce au regard de l'objectif de cohésion économique et sociale. Cependant, le fait qu'une entreprise en difficulté opère dans ces régions n'empêche pas 1 'application des critères généraux applicables à cette catégorie d'aide, à savoir le retour à la viabilité, la prévention de distorsions de concurrence indues, la proportionnalité aux coûts et avantages de la restructuration, la mise en œuvre complète du plan de restructuration et le respect des conditions imposées.

73. Loi n 95-115 du 4 février 1995, JORF du 5 février 1995.

${ }^{74}$ La Commission avait déjà examiné, en 1984, le versement pour la France, de primes d'aménagement du territoire que le gouvernement envisageait d'octroyer en faveur de projets industriels dans plusieurs départements (Bull. 1984. 10, point $\left.\mathrm{n}^{\circ} 2.1 .45\right)$.

${ }^{75}$. Pour les aides accordées au Pays basque à la Papeeiera Espanola, JOCE $\mathrm{n}^{\circ} \mathrm{C} 123$ du 5 mai 1993. 
Le gouvernement britannique avait estimé que le subventionnement des opérations de la CGM le protégeait des lois du marché en ce qui concerne les lignes des Antilles françaises, la CGM ayant pratiqué des tarifs en dessous des frais d'exploitation afin d'éliminer la concurrence ${ }^{76}$. La Commission rappelle, dans la logique des lignes directrices précitées, que l'aide à la restructuration doit simplement avoir pour fonction la viabilisation à long terme de l'entreprise bénéficiaire en évitant les distorsions indésirables de la concurrence, le respect du principe de proportionnalité étant essentiel. Elle a inséré, parmi les critères d'appréciation de 1'objectif assigné, l'abandon des lignes déficitaires, des coûts d'emploi des gens de la mer, le retour anticipé des chargements de banane sur la ligne des Antilles et la réduction du niveau d'endettement ${ }^{77}$. Il ne faut pas oublier que les principales activités de la CCM concerneraient les lignes sur les Antilles françaises avec le commerce de la banane et les intérêts outre-mer français (DOM/TOM), y compris les îles de l'océan Indien et celles du Pacifique. L'aide à la restructuration ne devant pas permettre au bénéficiaire de développer des capacités de production au cours de la mise en œuvre du plan de restructuration, la Commission n'a pas eu la preuve de l'existence d'une capacité excessive structurelle sur les lignes exploitées par la CGM. Elle ne doit pas non plus être utilisée en vue de donner au bénéficiaire les moyens d'accéder à de nouveaux marchés ou à des activités déficitaires. La participation de l'entreprise à sa propre réhabilitation est aussi un élément important à prendre en compte comme l'utilisation du produit de la cession des actifs pour soutenir la restructuration. Il en est de même du contexte (phase de privatisation) et de la trésorerie.

76. Déc. Commission, 17 juillet 1996 concernant l'aide à la Compagnie Générale Maritime dans le cadre d'un plan de restructuration, Aff. 97/14/CE, point n ${ }^{\circ} \mathrm{v}, \mathrm{i} ; \mathrm{n}^{\circ} \mathrm{L}$. 5 du 9 janvier 1997, p. 40.

77. Point $n^{\circ} \mathrm{IX}, \mathrm{i}$. 


\section{$3^{\circ}$ - La fiscalité particulière}

S'il est vrai que l'octroi de mer, ancien régime, constitue une taxe d'effet équivalent à un droit de douane, il entre aussi dans la catégorie des aides pour deux raisons, l'exonération fiscale au bénéfice de la production locale et l'affectation du produit de la taxe "à des fonds régionaux favorisant la production locale $", 78$. Le nouveau régime de l'octroi de mer résultant de la loi du 17 juillet 1992 n'a pas changé, à ce propos, la problématique malgré le réaménagement opéré. Par ailleurs, la suppression des exonérations fiscales au profit de la production locale, conformément au principe de non-discrimination qui gouverne l'article 95 du Traité CE relatif aux impositions intérieures, s'accompagnerait de l'augmentation des coûts de la production locale, ce qui reviendrait en fait à axer les efforts sur la réduction des coûts de productivité. Les entreprises locales ne peuvent que très difficilement compenser en amont les effets d'une politique économique nondiscriminatoire en aval.

\section{B - Les comportements étatiques fixant le cadre d'action d'entreprises opérant dans les régions ultrapériphériques}

La jurisprudence communautaire, sur les mesures provenant de collectivités publiques intéressant les régions ultrapériphériques met en exergue deux hypothèses : l'autonomie des entreprises vis-à-vis d'une intervention étatique (1) et la légitimité de cette intervention avec la possible sanction des entreprises (2).

${ }^{78}$. Sur ce dernier aspect, v. Danielle Perrot, “ A propos de l'arrêt Legros, quelques réflexions sur le statut communautaire des départements français d'outre-mer", Rev. March. Comm. n 368, mai 1993, p. 427. 


\section{$1^{\circ}$ - L'autonomie d'une entreprised'une région ultra- périphérique vis-à-vis du comportement étatique}

Dans le secteur automobile, la Commission et le TPI ont eu à examiner le comportement de concessionnaires automobiles situés à la Martinique. La Société Someco, établie à Fort-de-France (Martinique) avait déposé plainte auprès de la Commission en raison de certaines pratiques des Sociétés CCIE, SIGAM, SAVA, SIDA et Auto GM, concessionnaires respectifs à la Martinique de Toyota, Nissan, Mazda, Honda et Mitsubishi ${ }^{79}$ et importateurs de ces marques dans l'île. La plainte était fondée sur les articles 30 et 85 au motif que les pratiques de l'administration française avaient pour objectif d'empêcher les importations parallèles par Someco de véhicules de certaines de ces marques et des véhicules coréens Hyundaï. La requête de Someco, pour autant qu'elle a trait à l'article 85 du traité, dénonçait l'existence d'une entente entre les concessionnaires à la Martinique de voitures des cinq mêmes marques dont le but serait de bloquer l'accès au marché des concessionnaires de voitures d'autres marques japonaises et coréennes et l'existence d'une entente entre concessionnaires des cinq marques japonaises précitées ayant pour objet la répartition entre eux d'un quota d'importation fixé par l'administration française ${ }^{80}$. Le secteur automobile ferait 1'objet, selon les autorités françaises, d'une régulation dont les modalités sont fixées par les autorités publiques alors que les importateurs ne disposeraient d'aucune autonomie dans la gestion de cette régulation. En l'espèce, les ententes litigieuses concerneraient les importateurs pour la France de véhicules de marque japonaise sus-indiqués et l'administration française, ces entreprises acceptant de limiter à $3 \%$ leur part cumulée du marché intérieur français d'automobiles, en contrepartie d'un engagement des autorités françaises selon lequel le parc de voitures d'origine japonaise leur serait réservé. Il existait aussi une entente entre ces entreprises dont l'objet est la répartition entre elles de leur part cumulée du marché. A

79. TPI 29 juin 1993, Aff T-7/92, Asia Motor France SA (II) c. a c./ Commissions point $\mathrm{n}^{\circ} 10$, Rec. 1993, p II-669.

${ }^{80}$. TPI 18 septembre 1996, Aff. T-387/94, Asia Motor (III) c./ Commission, point $\mathrm{n}^{\circ} 43$, Rec. 1996, p. II-961. 
côté de ces ententes, des accords ont été conclus d'une part entre concessionnaires de marques japonaises à la Martinique et l'administration française et d'autre part entre concessionnaires. Dans le premier des accords intéressant les concessionnaires de véhicules japonais à la Martinique, ceux-ci avaient accepté de limiter à $15 \%$ leur part de marché martiniquais d'automobiles en contrepartie d'une promesse que le parc automobile d'origine japonaise leur serait exclusivement réservé. Dans le second accord les intéressant, ils ont réparti leurs parts globales du marché martiniquais. Selon le TPI, malgré la répartition des $15 \%$ entre entreprises concessionnaires à la Martinique, ceux-ci pouvaient répartir comme ils l'entendaient le marché puisque le protocole d'accord reposait sur leur accord de volontés $^{81}$. Les modalités de répartition du volume des importations en Martinique entre 1987 et 1991 n'ayant pas changé, cette inertie constituait, selon le TPI, un indice sérieux de l'autonomie du comportement de ces concessionnaires martiniquais ${ }^{82}$. Le fait que le comportement anticoncurrentiel des importateurs accrédités ait été favorisé ou encouragé par les autorités françaises est sans influence sur 1 'applicabilité de l'article 85 du traité $\mathrm{CEE}^{83}$. Dans l'affaire Asia Motor III $^{84}$, le TPI a estimé que ce n'est pas parce que les autorités françaises auraient imposé un quota de $15 \%$ qu'il en résulte nécessairement une entente entre les cinq concessionnaires de marques bénéficiaires. Si l'accréditation ne doit pas être assimilée à un droit d'importer, l'impossibilité pour la société Someco de commercialiser à la Martinique des voitures des marques Daïhatsu, Isuzu, Suzuki et Subaru tient au fait que seuls cinq concessionnaires martiniquais ont été accrédités en France ${ }^{85}$. Les importateurs accrédités sont exclusivement compétents pour délivrer des certificats de conformité aux

81. Point $n^{\circ} 43$.

${ }^{82}$. Point $\mathrm{n}^{\circ} 44$.

${ }^{83}$ Point $n^{\circ} 71$. En effet, si une mesure étatique ne prend pas les éléments d'une entente entre opérateurs ou est prise en consultation et avec l'accord des opérateurs concernés, les opérateurs ne sauraient invoquer la nature contraignante de la réglementation pour échapper à l'article 85 § I (CJCE 30 avril 1986, Aff. 209/84 à 213/84, Asjes, Rec. 19869, Rec 1986,p. 1425, point $\left.n^{\circ} 17\right)$.

${ }^{84}$. Asia Motor III, préc.

85 . Point $n^{\circ} 75$. 
concessionnaires à la Martinique alors que l'obtention d'un certificat de conformité est une condition nécessaire à l'immatriculation à la Martinique d'un véhicule importé ${ }^{86}$. Le TPI a conclu à l'absence de lien de causalité entre une éventuelle entente entre concessionnaires martiniquais et l'impossibilité pour une entreprise de vendre des véhicules d'autres marques que celles commercialisées par les concessionnaires et ce " indépendamment de la question de savoir s'il a été imposé unilatéralement par les autorités françaises ou s'il repose sur un accord conclu entre les cinq importateurs accrédités et les autorités françaises". Lorsqu'une disposition contraignante réglementaire susceptible de fausser le jeu de la concurrence à l'intérieur du marché commun ne présente aucun lien avec un comportement d'entreprises visé par l'article $85 \$ 1$, la simple observation des entreprises d'une telle disposition réglementaire échappe à l'application de l'article $85 \$ 1^{87}$, 1es opérateurs n'ayant pas de marge d'autonomie. En fait, les comportements d'Etat privilégiant certains opérateurs au détriment d'autres ont un effet anticoncurrentiel $^{88}$.

\section{$2^{\circ}$ - La légitimité de l'intervention étatique}

et la sanction possible d'entreprises

Le droit communautaire de la concurrence dans ces régions, même s'il reste embryonnaire et peu diffusé, s'impose à la mesure nationale ou régionale d'organisation du commerce dans les régions ultrapériphériques lorsque celle-ci intervient pour imposer ou favoriser des comportements anticoncurrentiels ou renforcer les effets d'une entente préexistante ${ }^{89}$. L'intervention de la mesure nationale est subordonnée à des contingences économiques et politiques. La

86. Point $n^{\circ} 76$.

${ }^{87}$. CJCE 17 novembre 1993, Aff. C-245/91, Ohra, Rec. 1993, p I-5851, point $\mathrm{n}^{\circ} 15$.

88 . Laurence Idot, Rev. Euope, novembre 1996, n 419, p. 19.

${ }^{89}$ CJCE 30 novembre 1995, Aff. C-134/94, Esso SA c./ Comunidad Autonoma de Canarias, point $n^{\circ} 19$, Rec. 1995, p. I-4223. 
recherche de la sécurité des approvisionnements tient compte de la situation des entreprises sur le territoire à approvisionner ${ }^{90}$ et du défaut de la production nationale ou locale, voire de risques de pénurie. L'intervention de l'Etat est légitime pour rég1ementer le développement des activités en raison du défaut de production locale.

Jusqu'à l'arrêt Esso SA c./Comunidad Autonoma de Canarias ${ }^{91}$, la Cour ne s'était pas prononcée, au regard du droit de la concurrence, sur les mesures nationales portant, dans un secteur de l'activité économique donné, organisation de l'approvisionnement à l'intérieur de l'une des régions ultramarines. En 1'espèce, le Conseil de 1'Industrie, du Commerce et de la Consommation des Iles Canaries a adopté une décision 54/1992 du 23 avril 1992 imposant désormais, par modification du décret 36/1991 du 14 mars 1991 portant approbation du règlement régissant les activités des grossistes en produits pétroliers dans les Iles Canaries, l'obligation de ravitaillement d'un minimum de quatre îles de l'archipel des Canaries. Déjà, la Cour a estimé que les mesures nationales concernant l'approvisionnement en produits pétroliers de l'Irlande pouvaient être justifiées par l'une des exigences importantes énoncées à l'article 36 du traité CE, à savoir la sécurité publique $^{92}$. Dans la lignée de la jurisprudence Campus Oil, l'Avocat Général Georgios Cosmas a justifié, sur le fondement de la sécurité publique, l'obligation d'approvisionnement d'un territoire déterminé constitutif d'une région archipélagique. Cependant, la différence entre ces deux affaires est notable puisqu'il était question, dans 1'arrêt Campus Oil, de maintenir une raffinerie publique en Irlande et de répondre aux besoins des importateurs alors que le litige Esso S.A. c./Comunidad Autonoma de Canarias est en étroite relation avec la volonté de pénétration d'une entreprise sur un marché local des produits pétroliers. Dans cette ultime affaire, la réglementation était de nature à exclure des opérateurs économiques exerçant les activités

${ }^{90}$. Dans l'arrêt Campus Oil (CJCE 10 juillet 1984, Aff. 72/83, Campus Oil c./ Ministre de l'Industrie et de l'Energie, Rec. 1984, p. 2727), des entreprises avaient fermé si bien que le gouvernement avait pris des mesures en vue du maintien d'une raffinerie publique.

91. CJCE 30 novembre 1995, Aff C-134/94, Esso SA c./ Comunidad Autonoma de Canarias, Rec. 1995, p. I-4223.

${ }^{92}$. CJCE 10 juillet 1984, Aff. 72/83, Campus Oil, Rec. 1984, p. 2727. 
mentionnées mais qui ne peuvent " techniquement ou financièrement" approvisionner un territoire déterminé tel que circonscrit par ladite réglementation. La violation de l'article $85 \mathrm{du}$ traité $C E$ ne pourrait résulter dans une telle hypothèse que de la collusion de volonté d'entreprises ayant des effets sur l'organisation de la distribution de produits dans ces régions. "L'article 85, lu en combinaison avec l'article 5, deuxième alinéa, ainsi que l'article 30 ne s'opposent pas à une réglementation, par laquelle les autorités régionales d'un Etat membre, responsables du gouvernement d'un archipel faisant partie de cet Etat, impose compte tenu des problèmes d'insularité, à tous les grossistes en produits pétroliers qui souhaitent étendre leurs activités à cette partie du territoire de l'État d'assurer le ravitaillement d'un nombre déterminé d'îles de l'archipel". On peut raisonnablement penser que l'approvisionnement minimal d'une région insulaire en énergie relève de l'intérêt général économique ${ }^{93}$. Lorsque les effets d'une législation nationale et ceux d'un comportement d'entreprise sont presque identiques, il faudrait considérer que tant qu'il n'est pas démontré que ladite réglementation est contraire au traité, le comportement est recevable sous réserve du fait que ce dernier serait de nature à dépasser les effets pratiques de la législation ${ }^{94}$. C'est dans ce cadre que le comportement des entreprises approvisionnant une partie du territoire des Canaries serait examiné.

$\mathrm{Au}$ total, le degré de la concurrence ne peut être que peu important dans les régions insulaires alors que, parallèlement, les entreprises locales doivent faire face à une concurrence accrue qu'accompagne actuellement le phénomène de mondialisation. Le souci local d'une protection de concurrence a suscité, dans le projet de traité d'Amsterdam une nouvelle rédaction de l'article $227 \quad \S 2$. L'alinéa 3 de ce paragraphe met l'accent sur la nécessité de prendre des mesures adaptées en matière douanière, fiscales, commerciale en précisant également l'intérêt des mesures prises dans la structure de la

93. Déc. Commission du 22 décembre 1992, Aff. IV/33.151, Jahrhundertvertrag et Aff. IV/33.997, VIK-GVSt ; J.O.C.E. $n^{\circ}$ L. 50 du 2 mars 1993, p. 14.

${ }^{94}$. TPI 18 novembre 1992, Aff. T-16/91, Rendo N.V. e. a. c./ Commission, point $\mathrm{n}^{\circ} 105$, Rec. 1992, p. II-2417. 
concurrence, en matière d'instauration de zones franches, $\mathrm{d}$ 'approvisionnement en matière première et en biens de consommation de première nécessité, d'aides d'Etat, d'accès aux fonds structurels et aux programmes horizontaux de la Communauté. Toutefois, l'ambivalence subsiste puisque l'alinéa 4 rappelle à la fois les spécificités et contraintes des régions ultrapériphériques et l'absence de compromission de l'intégrité et de la cohérence de l'ordre juridique communautaire. Sans s'arrêter aux ambiguïtés substantielles et procédurales que suggère la lecture de l'article $227 \S 2$ proposé, il ne faut pas oublier que l'alignement au droit commun est de droit lorsque l'on sait que, dans un cas d'espèce déterminé, la situation d'une région ultrapériphérique n'a pas été considérée comme "objectivement distincte de celle du reste de la Communauté $" 95$.

95. CJCE 26 mars 1987, Aff. 58/86, Coopérative Agricole d'Approvisionnement des Avirons c/ Direction des Douanes et Droits indirects de la Réunion, Rec. 1987, p. 1525 . 


\section{Résumé}

Les régions ultrapériphériques présentent l'originalité d'avoir une structure de marchés distincte du continent européen. Insulaires sauf la Guyane, petites, avec des productions différentes et un lien de dépendance économique fort par rapport à leur métropole respective, l'analyse structurelle a de ce fait une pertinence toute particulière. Bien sûr, il est délicat d'apprécier dans quelle mesure le droit communautaire de la concurrence s'applique à ces régions d'autant plus que la condition d'affectation du commerce entre les Etats membres doit être remplie. Les perspectives d'une application limitée ne sont bien sûr pas de nature à écarter le principe d'application récemment rappelé à propos des départements d'outre-mer dans l'arrêt Chevassus.

L'intérêt de cet article se situe également dans le fait que les dérogations découlant du droit communautaire de la concurrence peuvent être justifiées sur le fondement des critères de la différenciation. Ceux-ci sont pris en compte dans le cadre de ce droit en vertu $\mathrm{du}$ principe d'application immédiate dégagée à l'article 227 § 2 alinéa 1 du traité CE (avant le traité d'Amsterdam). Enfin, étant donné que l'intervention des Etats membres respectifs est intense, à quelque titre que ce soit, leur comportement doit être scrupuleusement appréhendé.

\section{Abstract}

The originality of the so-called ultra-peripheral regions lies in that their market structures are distinct from those of the European continent. Being small size islands (with the notable exception of La Guyane) with different productions and a strong link of economic dependence on their respective mainlands, they beautifully fit into the structural analysis model. Of course, it is not easy to assess how far EC laws on fair competition apply to those regions, the more so as the condition of trade affectation between member-states must be met. The outlook of limited implementation are not likely to set aside the principle of implementation itself as recently underscored with respect to the overseas departments in the Chevassus-Marche ruling .

The interest of the paper also lies in the fact that the dispensations provided for within the framework of the EC laws on fair competition can find their legal grounds in the differentiation criteria. Such criteria are taken into account under the immediate implementation clause as referred to in article 227 subsection 2 paragraph 1 of EC Treaty (before the Amsterdam Treaty). Last, owing to how much member-states intervene on whatever grounds, there is a necessity to anticipate their behavior the best one can.
Mots-clés - - - - - - - - - - - - - - -
Régions ultrapériphériques - Droit communautaire - Concurrence
$-\cdots-\cdots-\cdots-\cdots-\cdots-\cdots-\cdots$ Keys-words
Competition - EC Law - Ultra- peripheral regions

